\title{
Gluconeogenic signals regulate hepcidin gene expression via a CRBN-KLF15 axis
}

\author{
Jeong-Rang Jo ${ }^{1,2, \#}$, Sung-Eun Lee ${ }^{3, \#}$, Seungwon An ${ }^{4}$, Balachandar Nedumaran ${ }^{5}$, Swati Ghosh ${ }^{6}$, Keun-Gyu Park $^{1,2} \mathcal{E}^{2}$ \\ Yong Deuk Kim ${ }^{1,2,3, *}$ \\ ${ }^{1}$ Research Institute of Aging and Metabolism, Kyungpook National University, Daegu 41566, ${ }^{2}$ Department of Internal Medicine, School of \\ Medicine, Kyungpook National University, Kyungpook National University Hospital, Daegu 41944, ${ }^{3}$ Department of Applied Biosciences, \\ Kyungpook National University, Daegu 41566, Korea, ${ }^{4}$ Department of Ophthalmology and Visual Sciences, University of Illinois at Chicago, \\ Chicago, Illinois 60612, ${ }^{5}$ Barbara Davis Center for Diabetes, School of Medicine, University of Colorado Anschutz Medical Campus, \\ Aurora, Colorado 80045, ${ }^{6}$ Department of Pediatrics, School of Medicine, University of Colorado Anschutz Medical Campus, Aurora, \\ Colorado 80045, USA
}

\begin{abstract}
Hepcidin (HAMP) is synthesized in the liver. It is a key ironregulatory hormone that controls systemic iron homeostasis. Cereblon (CRBN) and Kruppel-like factor 15 (KLF15) are known to regulate diverse physiological functions. In this study, we investigated the role of CRBN on hepatic hepcidin gene expression and production under gluconeogenic stimuli. Fasted mice as well as forskolin (FSK)- and glucagon (GLU)-treated mice had reduced serum iron levels but increased expression levels of hepatic Crbn and KIf15 and hepcidin secretion. MicroRNA (miRNA) expression analysis of fasted and Ad-Crbninfected mice revealed significant reduction of microRNA-639 (miR-639). Hepatic overexpression of Crbn elevated hepcidin expression and production along with KIf15 gene expression, whereas knockdown of Crbn and Klf15 markedly decreased FSK- and fasting-mediated induction of hepcidin gene expression and its biosynthesis in mouse livers and primary hepatocytes. Moreover, expression of KLF15 significantly increased the activity of hepcidin reporter gene. It was exclusively dependent on the KLF15-binding site identified within the hepcidin gene promoter. Overall, this study demonstrates that CRBN and KLF15 are novel mediators of gluconeogenic signal-induced hepcidin gene expression and production. Thus, CRBN and KLF15 might be novel potential therapeutic targets to intervene metabolic dysfunction. [BMB Reports 2021; 54(4): 221-226]
\end{abstract}

*Corresponding author. Tel: +82-53-950-7768; Fax: +82-53-953-7233; E-mail: ydkim94@knu.ac.kr

${ }^{\text {"}}$ These authors contributed equally to this work.

https://doi.org/10.5483/BMBRep.2021.54.4.215

Received 29 September 2020, Revised 9 November 2020, Accepted 10 March 2021

Keywords: Cereblon, Fasting, Gene expression, Hepcidin, Kruppel-like factor 15

\section{INTRODUCTION}

Hepcidin encoded by the HAMP gene is known to be responsible for iron homeostasis in various mammals (1). It has been demonstrated that targeted disruption of hepcidin in the liver can reiterate the hemochromatosis phenotype accompanied by increased plasma iron and massive parenchymal iron accumulation (2). Hepcidin-deficient mouse models appear to be resistant to tissue damage and dysfunction during pulmonary iron overload with normal expression of upstream stimulatory factor 2 (USF2) $(3,4)$. Ceruloplasmin known to be involved in iron metabolism is deregulated in hepcidin-deficient mice (5). Conversely, mouse models of hepcidin transgenic overexpression in the liver have demonstrated severe iron-deficiency anemia (6). Hepcidin can also prevent iron overload and improve erythropoiesis in a mouse model of $\beta$-thalassemia (7). Hepatic hepcidin production is increased due to infection, inflammation, and severe iron overload, whereas it is decreased by hypoxia, iron-deficiency anemia, erythropoietin, and growth factors (8). Our recent study has shown that b-cell translocation gene 2 (BTG2)-yin yang 1 (YY1)-hepcidin signaling network controls mammalian iron homeostasis in the liver (8). The aim of the present study was to aimed to identify novel pathways associated with iron metabolism and gluconeogenesis.

Cereblon (CRBN) is a 442-amino acid protein exhibiting multiple functions in human brains and other tissues. It is associated with memory and learning (9). CRBN is also as an important molecular target of thalidomide (an antiemetic agent)-mediated teratogenicity (10). CRBN is predominantly expressed in the brain and moderately expressed in diverse tissues. It is a component of an E3 ubiquitin ligase complex by interacting with damage-specific DNA-binding protein 1 (DDB1), cullin 4 (Cul4), regulator of cullins 1 (ROC1), and ring box protein 1 (RBX1). Moreover, CRBN can directly interact with large-conductance calcium-activated potassium channels $\left(\mathrm{BK}_{\mathrm{Ca}}\right)$, voltage-gated chloride channel (ClC)-2, ikaros zinc finger (IKZF)1, IKZF3, and AMP-activated protein kinase (AMPK) (9-11). It plays

ISSN: 1976-670X (electronic edition)

Copyright (C) 2021 by the The Korean Society for Biochemistry and Molecular Biology

(c) This is an open-access article distributed under the terms of the Creative Commons Attribution Non-Commercial License (http://creativecommons.org/licenses/by-nc/4.0) which permits unrestricted non-commercial use, distribution, and reproduction in any medium, provided the original work is properly cited. 
a vital role in the regulation of ion transport and negatively modulates the AMPK signaling pathway in vivo and in vitro (12) and its metabolism (13). Studies have shown that Crbndeficient mice have hyperphosphorylated AMPK in their livers when fed with a normal diet. These mice are significantly protected against weight gain when they are fed with a high-fat diet. They also display an improved insulin sensitivity $(13,14)$. A recent study has reported that CRBN can inhibit the activation of AMPK both in vivo and in vitro by direct binding to the AMPK subunit (12). AMPK is an energy sensor at the cellular level by increasing ATP-producing catabolic pathways and suppressing ATP-consuming anabolic pathways (15). CRBN can regulate AMPK in vitro, and Crbn deficiency can preserve metabolic homeostasis by enhancing AMPK activity in alcoholic liver disease (16)

Kruppel-like factor 15 (KLF15) is a member of the KLF transcription factor family. It contains a zinc finger DNA-binding domain. KLF15 is expressed in diverse tissues such as the liver, kidney, pancreas, muscle, and heart (17). It is upregulated by glucagon and glucocorticoids during starvation or under diabetic conditions but downregulated by feeding and insulin $(18,19)$. KLFs are also involved in fibrosis, obesity, cardiovascular disease, cancer, and inflammatory conditions. KLF15 is a key positive regulator of gluconeogenesis but a negative regulator of cardiac hypertrophy and fibrosis $(18,20)$. However, the critical role of KLF15 in the regulation of iron metabolism through hepcidin gene expression remains unexplored.

In this study, we demonstrated that fasting and FSK treatment could significantly elevate hepatic hepcidin gene expression and production by increasing the expression of CRBN and KLF15. Moreover, disruption of CRBN and KLF15 markedly decreased hepcidin gene expression, eventually decreasing hepcidin secretion under FSK treatment. Our findings suggest that CRBN and KLF15 are mediators of fasting-induced hepatic hepcidin expression and its biosynthesis. Therefore, targeting CRBN and KLF15 might be a therapeutically important strategy to combat metabolic dysfunction.

\section{RESULTS}

\section{Fasting state and forskolin treatment increase hepatic hepcidin metabolism}

Gluconeogenic stimuli are known to regulate iron metabolism through hepcidin induction in starved mice (21). Cereblon (CRBN) deficiency is known to prevent diet-induced obesity and insulin resistance by increasing AMPK activation (13). We analyzed the expression of genes in livers of fasted mice to explore the link of Crbn, Klf15, and hepcidin under a fasting state. We observed that expression levels of hepatic CRBN, KLF15, and HAMP genes were significantly increased in fasted mice group than in non-fasted mice (Fig. 1A). Furthermore, we evaluated the expression of microRNAs during the fasting state. MicroRNA-639 (miR-639) expression was specifically decreased in fasted mice, whereas expression levels of miR-204
A

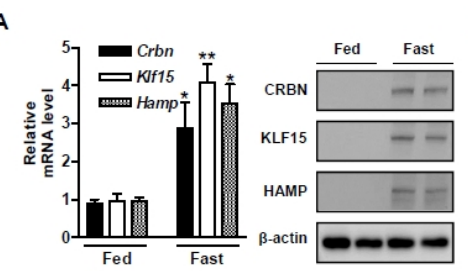

B

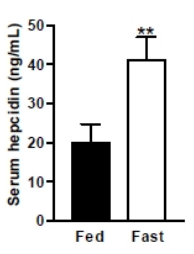

F

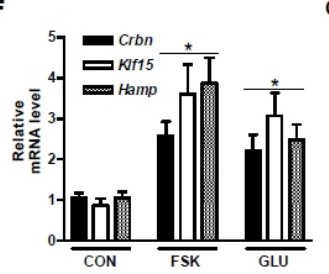

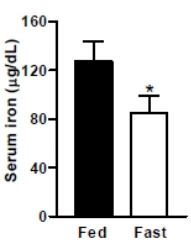
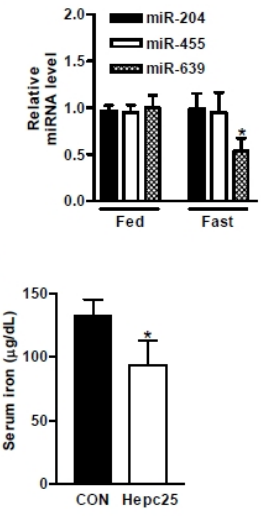

H

Fig. 1. Fasting and forskolin increases hepatic hepcidin gene expression and production in mice. (A) Wild-type (WT) mice were fed ad libitum (Fed) or fasted for $24 \mathrm{hr}$ (Fast). Gene expressions were analyzed by qPCR using gene-specific primers. Expression levels of various proteins in liver tissue extracts were analyzed by immunoblotting using specific antibodies. (B) Expression profiles of predicted microRNAs (miR) in mouse livers. (C) Serum hepcidin and (D) iron contents in indicated mice groups. (E) Serum iron concentration after hepcidin-25 (Hepc25, $100 \mu \mathrm{g} / \mathrm{kg}$ ) treatment for $12 \mathrm{~h}$. (F) WT mice were injected intraperitoneally with forskolin (FSK, $5 \mathrm{mg} / \mathrm{kg}$ ) and glucagon (GLU, $10 \mu \mathrm{g} / \mathrm{kg}$ ) for $6 \mathrm{hr}$. qPCR experiments were then performed using various primers. (G) Serum levels of hepcidin and $(H)$ iron at the indicated condition ( $n=5$ mice per group). ${ }^{*} P<0.05, * * P<0.01$ vs. fed mice or untreated control mice.

and miR-455 were unchanged in fasted mice (Fig. 1B). Overall, these results suggest that CRBN and miR-639 play a key role in the regulation of hepatic hepcidin gene expression under a fasting state. Consistent with Hamp gene expression, serum hepcidin levels were also significantly elevated in fasted mice than in mice at a fed state (Fig. 1C), indicating a role of hepcidin during gluconeogenesis. Next, we explored hepcidin expression in response to gluconeogenic stimuli (forskolin and glucagon) and found that forskolin (FSK) and glucagon (GLU) treatment increased expression levels of Crbn, Klf15, and Hamp in mouse livers (Fig. 1F). Similarly, FSK and GLU exposure elevated serum hepcidin levels compared to the control groups (Fig. 1G). However, serum iron levels were markedly reduced in mic of fasted, Hepc25-treated, FSK-treated, or GLUtreated group than in their respective control group (Fig. 1D, E, $\mathrm{H})$. Taken together, these results suggest an important link between CRBN and hepcidin in the regulation of iron metabolism in response to gluconeogenic stimuli. 


\section{CRBN increases hepatic hepcidin gene expression}

We investigated the crucial role of Crbn as a key positive regulator of hepcidin gene expression. Using an adenoviral delivery system expressing $\mathrm{Crbn}$ (Ad-Crbn) or a control green fluorescent protein (Ad-GFP), we observed that Crbn was ectopically expressed in primary mouse hepatocytes. Ad-Crbn was successfully overexpressed in primary mouse hepatocytes. Transduction of Ad-Crbn significantly enhanced both mRNA and protein levels of KLF15 and HAMP compared to those in Ad-GFP control groups (Fig. 2A, B). Lenalidomide-induced CRBN is known to regulate argonaute 2 (AGO2) protein and its target microRNAs in multiple myeloma cells (22). Hence, we sought to investigate whether Crbn might have any role in the regulation of microRNAs in mouse livers. MiR-639 expression level was specifically decreased by the administration of Ad-Crbn, whereas miR-204 and miR-455 expression levels were not changed in Ad-Crbn-infected mice (Fig. 2C). Overall, these results suggest that CRBN can control hepatic hepcidin gene expression via the upregulation of KLF15 and the downregulation of miR-639. Conversely, we examined whether Crbn knockdown could modulate FSK- and fasting-mediated induction of KIf15 and hepcidin metabolism in primary mouse hepatocytes and mouse livers using an adenoviral knockdown method. As shown in Fig. 2D-F, expression levels of CRBN, KLF15, and HAMP genes were significantly upregulated by FSK treatment and fasting state, and this phenomenon was markedly reduced by Crbn knockdown. Interestingly, the increase in serum hepcidin level induced by fasting was decreased by silencing of Crbn, but not in serum iron level (Fig. 2G). Collectively, these findings suggest that CRBN can mediate the induction of hepcidin gene by FSK treatment.

\section{KLF15 regulates hepcidin gene expression}

To understand effects of KLF15 on hepcidin gene expression and production, we modulated hepatic hepcidin gene expression using an adenoviral delivery system in primary mouse hepatocytes. As shown in Fig. 3A, Klf15 was successfully delivered into primary mouse hepatocytes and also significantly elevated Hamp gene expression. Especially, overexpression of Ad-KIf15 increased hepcidin amounts compared to those in the control groups (Fig. 3B). We further investigated a more direct effect of KLF15 on FSK- and fasting-induced hepcidin gene expression and production using adenovirus-mediated silencing of Klf15 (Ad-shKlf15) both in vivo and in vitro. Expression levels of Klf15 in control and FSK-treated primary mouse hepatocytes were effectively repressed by Ad-shKIf15. FSK- and fastingmediated induction of Hamp gene expression was dramatically attenuated by Klf15 knockdown (Fig. 3C, E). Consistent with Hamp gene expression, FSK- and fasting-induced serum hepcidin amounts were prominently decreased by knockdown of endogenous Klf15, but not serum iron levels (Fig. 3D, F). Overall, these observations strongly suggest that KLF15 can regulate hepatic hepcidin gene expression and production.
A

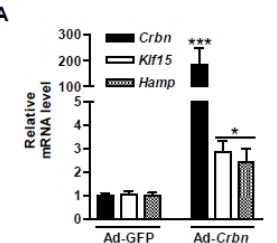

B
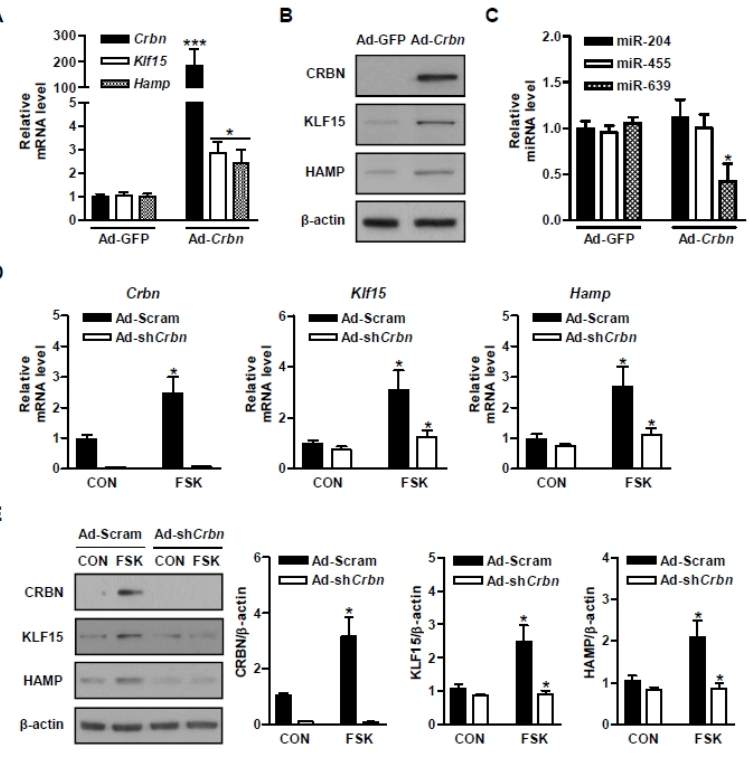

$\mathbf{F}$

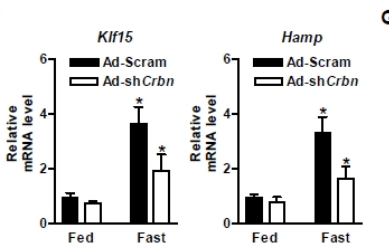

G
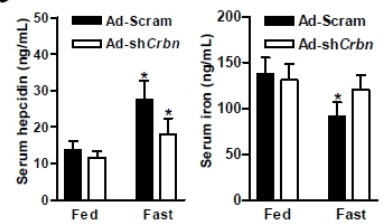

Fig. 2. CRBN elevates hepatic hepcidin gene expression in mouse primary hepatocytes. (A) Mouse primary hepatocytes were infected with Ad-GFP and Ad-Crbn at a multiplicity of infection of 60 for $36 \mathrm{hr}$. Gene expression was analyzed by qPCR using various primers. (B) Expression levels of various proteins in mouse primary hepatocytes were analyzed by immunoblotting using corresponding antibodies. (C) Expression levels of miR-204, miR-455, and miR-639 were quantified by qPCR analysis. (D) Mouse primary hepatocytes were infected with Ad-Scramble (Ad-Scram) and Ad-shCrbn at a multiplicity of infection of 60 for $36 \mathrm{hr}$. After infection, cells were treated with FSK $(10 \mu \mathrm{M})$ for $12 \mathrm{hr}$. Total RNAs were utilized for qPCR analysis using gene-specific primers. (E) Whole-cell extracts were harvested from indicated groups of mouse primary hepatocytes and immunoblotted with specific antibodies. (F) WT mice were infected with Ad-Scram and Ad-shCrbn for 7 days. These mice were then fasted for $24 \mathrm{hr}$. Total RNAs were utilized for qPCR analysis using gene-specific primers. (G) Serum levels of hepcidin and iron at the indicated condition. $* P<0.05, * * P<0.01$, and $* * * P<$ 0.001 vs. untreated control cells, Ad-GFP, or FSK-treated cells, fed, or fasted mice.

\section{KLF15 is recruited to the hepcidin promoter}

Next, we explored whether KLF15 could directly control the transcriptional activity of hepcidin induced by FSK treatment. We observed that FSK treatment or transiently expressed Klf15 significantly elevated Hamp promoter activity (Fig. 4A). Through in silico analysis, we predicted a KLF15-binding site on the Hamp promoter. We then mutated this binding site on the Hamp promoter. A reporter gene assay indicated that FSK treatment and Klf15 transfection increased the Hamp promoter 
A
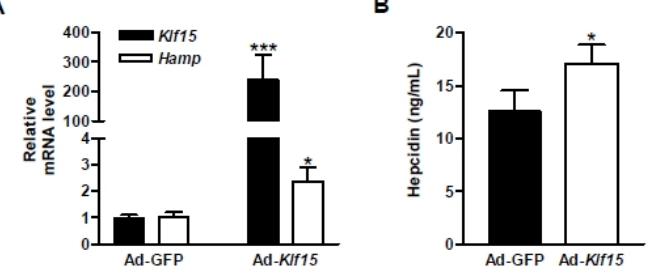

D

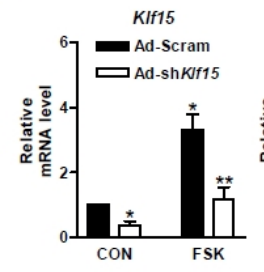

E

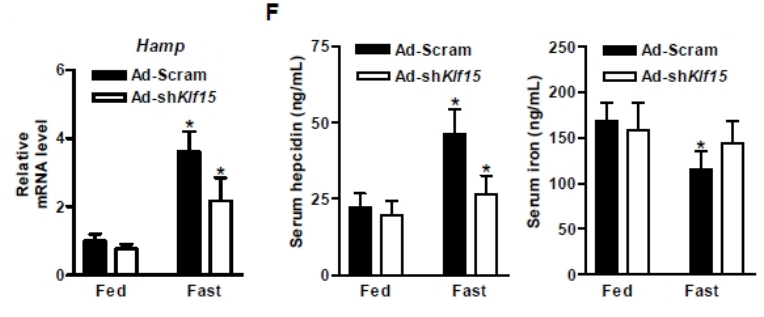

Fig. 3. KLF15 regulates hepatic hepcidin gene expression and production in mouse primary hepatocytes. (A) Mouse primary hepatocytes were infected with Ad-GFP and Ad-Klf15 at a multiplicity of infection of 60 for $36 \mathrm{hr}$. Gene expressions were analyzed by qPCR using indicated primers. (B) Hepcidin concentrations in observed mouse primary hepatocytes. (C) Mouse primary hepatocytes were infected with Ad-Scram and Ad-shKlf15 at a multiplicity of infection of 60 for $36 \mathrm{hr}$ and then treated with FSK for $12 \mathrm{hr}$. Total RNAs were utilized for qPCR analysis using gene-specific primers. (D) Hepcidin concentrations under indicated conditions. (E) WT mice were infected with Ad-Scram and Ad-shKlf15 for 7 days. These mice were then fasted for $24 \mathrm{hr}$. Quantitative PCR analysis was performed to determine Hamp expression in the liver. (F) Serum levels of hepcidin and iron under the observed condition. $* \mathrm{P}<$ $0.05, * * \mathrm{P}<0.01$, and $* * * \mathrm{P}<0.001$ vs. untreated controls, Ad-GFP, FSK-treated cells, fed, or fasted mice.

activity, whereas this phenomenon was strikingly abolished in the group with KLF15-binding site-mutated (mt) Hamp gene promoter compared to the group with the wild-type (wt) promoter (Fig. 4B). To further examine the recruitment of KLF15 protein to the Hamp gene promoter and the effect of CRBN on KLF15 recruitment, we performed chromatin immunoprecipitation (ChIP) assay using KLF15 antibody in primary mouse hepatocytes. There was no recruitment of KLF15 protein to the distal (Dis) region of the promoter where we did not find any KLF15binding site from the in silico analysis. As anticipated, endogenous KLF15 occupancy on the proximal (Pro) region with the KLF15-binding site was significantly increased by FSK treatment compared to that in control groups (Fig. 4C). Overall, these results strongly suggest that KLF15 is recruited
A

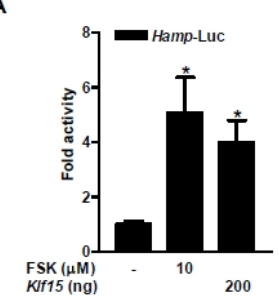

B

Hamp (wt) - 370 GCACCCT -364 Hamp (mt) $-370 \mathrm{GC} \mathrm{tt} \mathrm{t} \mathrm{CT}-364$ ${ }^{9} 7 \mathrm{Camp-Luc(wt)}$

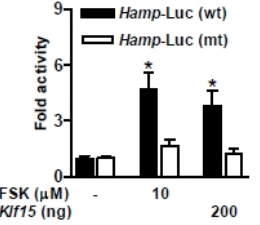

C

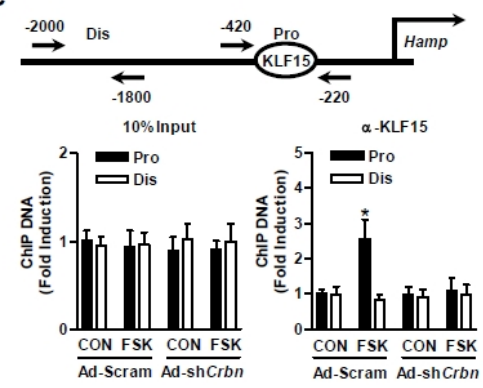
D

Fig. 4. KLF15 is an important regulator of hepcidin gene transcription in hepatocytes. (A) AML-12 cells were transiently transfected with Klf15 and the indicated reporter gene for $36 \mathrm{hr}$ and then treated with FSK for $12 \mathrm{hr}$. (B) AML-12 cells were co-transfected with wild-type (wt) and mutant (mt) forms of the Hamp gene promoter and Klf15 for $36 \mathrm{hr}$. After transfection, cells were treated with FSK for 12 hr. (C) Chromatin immunoprecipitation (ChIP) assay for the recruitment of KLF15 on the Hamp gene promoter. Mouse primary hepatocytes were infected with Ad-Scram and Ad-shKlf15 for $36 \mathrm{hr}$ and then treated with FSK for $12 \mathrm{hr}$. Cell lysates were immunoprecipitated with an anti-KLF15 antibody. Purified DNA samples were employed for PCR using specific primers binding to specific proximal (Pro) and nonspecific distal (Dis) regions on the Hamp gene promoter. Input was $10 \%$ of soluble chromatin. (D) A schematic model illustrating the regulation of hepcidin gene expression and production. Fasting state and forskolin treatment can enhance hepatic hepcidin gene expression by stimulating the CRBN-KLF15 signaling network, which subsequently controls iron metabolism via an increase of hepcidin output and a decrease of iron production. ${ }^{*} P<0.05$ vs. untreated control.

to the hepcidin gene promoter region to enhance the transcription of hepcidin gene.

\section{DISCUSSION}

Using in vivo and in vitro experiments, we demonstrated that CRBN enhanced hepatic hepcidin gene expression and its secretion by upregulating KLF15 expression. We found that gluconeogenic signals remarkably induced the transcription and production of the hepatic hepcidin gene by stimulating the CRBN-KLF15 signaling pathway. In contrast, stimulatory effects of fasting condition or FSK treatment on hepatic hepcidin gene expression and its secretion were markedly decreased by the knockdown of endogenous Crbn and Klf15 in primary mouse hepatocytes. These findings indicate that the induction of hepatic hepcidin production by the fasting state is KLF15- 
dependent. Our results suggest that the gluconeogenic stimuliCRBN-KLF15 signaling network manifests a potent molecular mechanism underlying the regulation of hepcidin gene expression and production under both in vivo and in vitro conditions.

Previous studies have reported that gluconeogenic signals can modulate hepatic hepcidin gene expression and its biosynthesis in mice $(21,23,24)$. Hepcidin is an established modulator of iron metabolism, inflammation, hypoxia, and erythropoiesis $(25,26)$. During fasting conditions, the BTG2-KLF15 signaling network can modulate the biosynthesis of hepatic fibroblast growth factor 21 (17). Our previous study has shown that AMPK is negatively regulated by CRBN in mice with alcoholic liver disease (16). However, there is no information regarding the potential role of CRBN in modulating hepatic hepcidin gene expression or its secretion. Our current results demonstrated that elevated CRBN expression by the fasting state or FSK treatment could control the production of hepatic hepcidin by promoting KIf15 expression. The fasting state and FSK challenge enhanced expression levels of hepatic Crbn, Klf15, and Hamp genes and increased serum hepcidin levels in mice, but not iron levels (Fig. 1). Transduction of Ad-Crbn efficiently elevated hepatic hepcidin gene expression and production via the induction of KLF15 expression, whereas this phenomenon was prominently abolished in Crbn silenced cells (Fig. 2). These findings suggest that CRBN plays a vital role in regulating fasting- and FSK-induced hepatic hepcidin gene expression and production by inducing KLF15 expression.

Glucagon and glucocorticoids are known to induce the transcription factor KLF15 during a fasting state, whereas insulin is known to downregulate KLF15 expression under a fed condition (18-20). It is known that KLF15 can modulate fibrosis, obesity, cardiovascular disease, cancer, and inflammatory conditions. Based on this information, we further characterized the novel molecular mechanisms underlying the fasting-mediated induction of hepcidin gene transcription by the CRBN-KLF15 signaling pathway in hepatocytes. As depicted in Fig. 3, KLF15 positively regulated hepcidin gene expression and production in primary mouse hepatocytes during FSK treatment. For the first time, we identified a KLF15-binding site on the Hamp gene promoter. We also found that the Hamp promoter activity was efficiently increased by a transient transfection of Klf15 (Fig. 4). Overall, our results suggest that the CRBN-KLF15 signaling pathway can mediate the induction of hepcidin gene by gluconeogenic signals.

$\mathrm{Xu}$ et al. have demonstrated that lenalidomide-induced cell growth and survival are involved in the regulation of CRBNdownstream binding protein AGO2, an essential protein for microRNA maturation. They found that lenalidomide could significantly induce CRBN, subsequently altering expression levels of both AGO2 protein and its target microRNAs in multiple myeloma cells (22). Our results demonstrated that miR-639 expression was specifically decreased in fasted mice. Moreover, miR-639 expression was exclusively decreased by the administration of Ad-Crbn (Figs. 1 and 2). Interestingly, Ad-
Crbn-induced expression levels of Klf15, phosphoenolpyruvate carboxykinase 1 (Pck1), and glucose-6 phosphatase (C6pc) genes were reduced by miR-639 mimics. However, an inhibitor of miR-639 did not alter the induction of Klf15 or gluconeogenic gene by Ad-Crbn. Collectively, these results strongly suggest that $\mathrm{CRBN}$-mediated downregulation of miR-639 can regulate hepatic gluconeogenic gene expression by inducing KLF15 expression in the liver and primary hepatocytes (data not shown). However, the molecular mechanism of CRBN-microRNAKLF15 signaling pathway remains unclear. It needs to be investigated in the future. Genetically engineered mouse models of Crbn and Klf15 genes would be useful for exploring their physiological roles in the liver in relation to metabolic dysfunction. High-throughput sequencing analysis of these models would also be valuable to discover various regulators involving $\mathrm{CRBN}$-downstream binding protein $\mathrm{AGO} 2$ and microRNAs linked to CRBN-AGO2-KLF15 and/or the CRBN-microRNA-KLF15 signaling network.

Hepcidin is produced due to iron loading and inflammation at a high rate in the liver. It is cleared from the circulation by kidneys to maintain iron balance in the body $(1,7,27)$. Hepcidin deficiency can result in iron overload $(3-5,28)$, whereas overloading of hepcidin can lead to the development of iron-restrictive anemia, autoimmune inflammatory disorders, chronic kidney disease, cancers, infections, and inherited ironrefractory iron-deficiency anemia $(25,27,28)$. Psychological stress and overload exercise can reduce serum iron levels and result in the inhibition of erythropoiesis in rats $(29,30)$. All these studies have shown that short-term food deprivation and reduced iron levels could lead to psychological stress and depression symptoms in patients with anorexia or other eating disorders. Our results indicate that a fasting condition can increase hepcidin production but decrease iron levels. Interestingly, hepcidin treatment strikingly reduced serum iron levels in mice (Fig. 1), indicating an inverse correlation between hepcidin and serum iron levels under a fasting condition. In addition, iron-mediated hepcidin regulation is associated with the bone morphogenetic protein (BMP)-SMAD pathway, whereas inflammation-mediated regulation is associated with both the interleukin-6 (IL-6)/Janus kinase (JAK)/signal transducer and activator of transcription signaling axis and the BMP-SMAD pathway $(1,7,25,26,31)$. Increased expression of hepcidin regulation under a fasting state can lead to decreased serum iron levels via the upregulation of CRBN, KLF15, and HAMP. Therefore, altering the serum hepcidin rate can upregulate its circulating concentration in a fasting state, consequently changing serum iron flow.

In conclusion, our current study demonstrates that hepcidin is a possible novel target of CRBN and that CRBN encourages hepatic hepcidin gene expression and production by inducing KLF15 expression when exposed to gluconeogenic signals such as a fasting state or FSK treatment. We speculate that the increase in CRBN expression induced by gluconeogenic stimuli can efficiently modulate hepatic hepcidin metabolism by 
enhancing the expression of KLF15. Furthermore, upregulated CRBN can decrease serum iron levels under a fasting state (Fig. 4D). This novel molecular mechanism involving hepatic hepcidin metabolism by the CRBN-KLF15 signaling network might provide a better understanding to develop potential therapeutic agents and important strategies to intervene metabolic dysfunction caused by iron-deficiency anemia.

\section{MATERIALS AND METHODS}

Additional detailed methods are described in Supplementary information.

\section{ACKNOWLEDGEMENTS}

This research was supported by Kyungpook National University Development Project Research Fund, 2018 (to Y.D.K.).

\section{CONFLICTS OF INTEREST}

The authors have no conflicting interests.

\section{REFERENCES}

1. Anderson ER and Shah YM (2013) Iron homeostasis in the liver. Compr Physiol 3, 315-330

2. Zumerle S, Mathieu JR, Delga S et al (2014) Targeted disruption of hepcidin in the liver recapitulates the hemochromatotic phenotype. Blood 123, 3646-3650

3. Lesbordes-Brion JC, Viatte L, Bennoun M et al (2006) Targeted disruption of the hepcidin 1 gene results in severe hemochromatosis. Blood 108, 1402-1405

4. Deschemin JC, Mathieu JRR, Zumerle S, Peyssonnaux C and Vaulont S (2017) Pulmonary iron homeostasis in hepcidin knockout mice. Front Physiol 8, 804

5. Viatte L, Lesbordes-Brion JC, Lou DQ et al (2005) Deregulation of proteins involved in iron metabolism in hepcidin-deficient mice. Blood 105, 4861-4864

6. Nicolas G, Bennoun M, Porteu A et al (2002) Severe iron deficiency anemia in transgenic mice expressing liver hepcidin. Proc Natl Acad Sci U S A 99, 4596-4601

7. Ganz T and Nemeth E (2012) Hepcidin and iron homeostasis. Biochim Biophys Acta 1823, 1434-1443

8. Lee SE, Hwang SL, Jang WG, Chang HW and Kim YD (2015) B-cell translocation gene 2 promotes hepatic hepcidin production via induction of Yin Yang 1. Biochem Biophys Res Commun 460, 996-1001

9. Shi Q and Chen L (2017) Cereblon: a protein crucial to the multiple functions of immunomodulatory drugs as well as cell metabolism and disease generation. J Immunol Res 2017, 9130608

10. Chang XB and Stewart AK (2011) What is the functional role of the thalidomide binding protein cereblon? Int J Biochem Mol Biol 2, 287-294

11. Kim HK, Ko TH, Nyamaa B et al (2016) Cereblon in health and disease. Pflugers Arch 468, 1299-1309

12. Lee KM, Yang SJ, Choi JH and Park CS (2014) Functional effects of a pathogenic mutation in Cereblon (CRBN) on the regulation of protein synthesis via the AMPK-mTOR cascade. J Biol Chem 289, 23343-23352

13. Lee KM, Yang SJ, Kim YD et al (2013) Disruption of the cereblon gene enhances hepatic AMPK activity and prevents high-fat diet-induced obesity and insulin resistance in mice. Diabetes 62, 855-1864

14. Lee KM, Jo S, Kim H, Lee J and Park CS (2011) Functional modulation of AMP-activated protein kinase by cereblon. Biochim Biophys Acta 1813, 448-455

15. Foretz $M$ and Viollet B (2011) Regulation of hepatic metabolism by AMPK. J Hepatol 54, 827-829

16. Kim YD, Lee KM, Hwang SL et al (2015) Inhibition of cereblon by fenofibrate ameliorates alcoholic liver disease by enhancing AMPK. Biochim Biophys Acta 1852, 26622670

17. Kim YD, Hwang SL, Jeon HJ et al (2019) B-cell translocation gene 2 enhances fibroblast growth factor 21 production by inducing Kruppel-like factor 15 . Sci Rep 9, 3730

18. McConnell BB and Yang VW (2010) Mammalian Kruppellike factors in health and diseases. Physiol Rev 90, 13371381

19. Teshigawara K, Ogawa W, Mori T et al (2005) Role of Kruppel-like factor 15 in PEPCK gene expression in the liver. Biochem Biophys Res Commun 327, 920-926

20. Pearson R, Fleetwood J, Eaton S, Crossley M and Bao S (2008) Kruppel-like transcription factors: a functional family. Int J Biochem Cell Biol 40, 1996-2001

21. Vecchi C, Montosi G, Garuti C et al (2014) Gluconeogenic signals regulate iron homeostasis via hepcidin in mice. Gastroenterology 146, 1060-1069

22. Xu Q, Hou YX, Langlais P et al (2016) Expression of the cereblon binding protein argonaute 2 plays an important role for multiple myeloma cell growth and survival. BMC Cancer 16, 297

23. Zhang $Y$, Wang $X$, Wu Q et al (2018) Adenine alleviates iron overload by cAMP/PKA mediated hepatic hepcidin in mice. J Cell Physiol 233, 7268-7278

24. Aigner E, Felder TK, Oberkofler H et al (2013) Glucose acts as a regulator of serum iron by increasing serum hepcidin concentrations. J Nutr Biochem 24, 112-117

25. Ruchala P and Nemeth E (2014) The pathophysiology and pharmacology of hepcidin. Trends Pharmacol Sci 35, 155161

26. Rishi G, Wallace DF and Subramaniam VN (2015) Hepcidin: regulation of the master iron regulator. Biosci Rep 35, e00192

27. Fung E and Nemeth E (2013) Manipulation of the hepcidin pathway for therapeutic purposes. Haematologica $98,1667-$ 1676

28. Ganz T and Nemeth E (2011) Hepcidin and disorders of iron metabolism. Annu Rev Med 62, 347-360

29. Wei C, Zhou J, Huang X and Li M (2008) Effects of psychological stress on serum iron and erythropoiesis. Int J Hematol 88, 52-56

30. Navas FJ and Córdova A (2000) Iron distribution in different tissues in rats following exercise. Biol Trace Elem Res 73, 259-268

31. Sangkhae $V$ and Nemeth (2017) Regulation of the iron homeostatic hormone hepcidin. Adv Nutr 8, 126-136 